\title{
POSTERS
}

doi: http://dx.doi.org/10.7124/bc.0009D0

\section{A-1. The nuclear thiol redox systems in cancer cells}

Malgorzata Adamiec, Dorota Hudy, Patryk Bil, Sylwia Ciesielska, Magdalena Skonieczna

Biosystems Group, Institute of Automatic Control, Silesian University of Technology, Akademicka 16, 44-100 Gliwice, Poland.

malgorzata.adamiec@polsl.pl

The goal of this work was to study the role of proteins with oxidizable thiols, from redox systems based on thioredoxin-1 (Trx-1) and glutathione (GSH), in HCT 116 (colorectal cancer) cells under oxidative stress. The widespread presence in cells of anti-oxidative proteins in their reduced electrochemical state serves as a physiological defense against different oxidative and damaging agents such as ultraviolet radiation (UV), ionizing radiation (IR), reactive oxygen species (ROS), reactive nitrogen species (RNS) etc. In different cellular compartments, cytoplasm, organelles and especially in the nucleus thiol/disulfide redox systems play roles of important controllers of chromatin stability, transcription, nuclear protein import and export, and DNA replication and repair processes [1]. A set of specific reductases (thioredoxin reductase, TrxRD, and glutathione reductase, GSR) convert oxidized TrxSS and GSSG to their monomer state again and thus control the thiol/ disulfide redox states. Different types of GSRs were reported in the nucleus [1]. Trx-1 in the nucleus responses mainly for redox state via the redox activity maintains conserved Cys residues of transcription factors, damaged DNA repair process, transcriptional factors activation cell death and survival signaling [1]. Methods: ROS and RNS levels were measured in HCT 116 cells at different time points $(1,3,6,12$ and 24h) after irradiation with UVA radiation at a dose of $10 \mathrm{~kJ} / \mathrm{m} 2$. The levels of transcripts for Trx 1 and TrxRD were assessed by real-time PCR. GSSH levels were estimated by Westen Blot with the BioGEE reagent specific for gluthationylated proteins and TrxRD transcript levels by real-time PCR. Cellular localization of GSH was studied by fluorescence imaging. Results: ROS and RNS levels had increased 3 and $12 \mathrm{~h}$ after exposure to UVA. This wave of radicals immediately activated thiols from redox systems, the level of GSH protein at $3 \mathrm{~h}$ and the level of transcripts for Trx 1 at 12 and 24h. Gluthationylated proteins were reduced after 24 $h$ by GSR reductase and the pool of GSH form recovered. Trx 1 was reduced efficiently by TrxRD reductase, with the highest level of transcripts $12 \mathrm{~h}$ after UV exposure. Conclusions: Trx1 is imported into the nucleus during oxidative stress, and increased ROS/ RNS levels activate oxidation of both Trx1 and GSH redox states in HCT116 cells after UV irradiation. Efficient reductase-based systems mediated by TrxRD and GSR recover the basal level of reduced/monomer (GSH) and oxidizable thiol (Trx1) molecules.

This work was supported by grant No. 02/010/BK_18/0102 from Silesian University of Technology in Gliwice, Poland.

References: 1. Go Y-M, Jones DP; 2010. Redox Control Systems in the Nucleus: Mechanisms and Functions. Antioxid Redox Signal. 13(4) 